Egyptian

Orthodontic Journal

\title{
Influence of Local Application of Simvastatin on Orthodontic Relapse: An Experimental Study
}

\author{
Hani AISwafeeri ${ }^{1}$, Walid ElKenany ${ }^{2}$, Mohamed Mowafy ${ }^{3}$, Sahar Karam ${ }^{4}$
}

\section{ABSTRACT:}

Introduction: Orthodontic relapse is a physiological response of the supporting tissues to force application threatening the success of orthodontic therapy. The objective of this study was to evaluate the influence of local application of simvastatin on the magnitude of orthodontic relapse. Methods: Orthodontic tooth movement was induced in 10 white New Zealand rab6its. After 21 days of active tooth movement, orthodontic appliances were removed and the experimental teet $h$ were allowed to relapse for further 21 days. During the relapse phase, one mandibular quadrant received local simvastatin administration while the corresponding mandibular quadrant received control veficle solution on a weekly basis. Magnitudes of experimental tooth movement and post-orthodontic relapse were calculated. Results: The mean relapse magnitude was $1.31 \pm 0.53 \mathrm{~mm}$ in the quadrant receiving control veficle solution and $1.14 \pm 0.47 \mathrm{~mm}$ in the quadrant receiving simvastatin administration. Neither the relapse magnitude nor the relapse percentage showed a significant difference between the two quadrants. Conclusion: Local simvastatin administration could not minimize post-orthodontic relapse magnitude to a significant level.

\section{INTRODUCTION}

Relapse is a physiological response of the supporting tissues to force application and has been a major challenging clinical issue with respect to the goals of successful orthodontic treatment. ${ }^{1,2}$ The classic approaches to orthodontic retention primarily involve the use of fixed or removable retainers whose caveats include the reliance on patient compliance and the long duration of retention required to ensure stability. ${ }^{3}$

Various systemically and locally administrated agents have been reported to reduce the amount of relapse in animal models, including bisphosphonate, ${ }^{4}$ osteoprotegerin, ${ }^{5}$ relaxin, ${ }^{6}$ and bone morphogenetic proteins., ${ }^{7}$ Although the mechanisms of action are different, relapse is decreased by modification of the remodeling process of the dental supporting tissues. These findings prompted the possibility of safe pharmacotherapeutic strategies designed to manipulate alveolar bone remodeling to minimize post-orthodontic relapse.

A hypolipidemic agent, simvastatin, has recently been expected to serve as a therapeutic agent for osteoporosis. These drugs, known as statins, represent a new approach for treating osteoporosis, one which emphasizes building new bone to replace bone that has already

\footnotetext{
1- Assistant Lecturer, Department of Orthodontics, Faculty of Dentistry, Pharos University, Egypt

2- Professor, Department of Orthodontics, Faculty of Dentistry, Alexandria University, Egypt

3- Associate Professor, Department of Orthodontics, Faculty of Dentistry, Alexandria University, Egypt

4-Professor, Department of Oral Biology, Faculty of Dentistry, Alexandria University, Egypt
} 
deteriorate ${ }^{8}$ Out of 30,000 natural compounds, Mundy and his colleagues ${ }^{9}$ identified statins as the only natural product that specifically increased bone morphogenetic protein-2 (BMP-2) gene expression in osteoblasts and increased levels of bone formation proteins in these cells. There was also a striking increase in osteoblast cell numbers after statin application since BMP-2 is one of the most potent growth factors targeting bone formation in vivo, which stimulates osteoblastic differentiation and proliferation. ${ }^{10}$

Inhibition of the 3-hydroxy-3-methylglutaryl-coenzyme-A reductase enzyme and the subsequent blockage of the mevalonate pathway is probably the most important mechanism of inhibition of bone resorption by simvastatin. ${ }^{11}$ Yazawa et al ${ }^{12}$ showed that relatively low concentrations of simvastatin promoted cell proliferation and osteoblastic differentiation. Lee et al ${ }^{13}$ observed increased bone formation and higher maximum force to fracture after local application of statins into rat mandibles. Ozec et al ${ }^{14}$ revealed that local application of simvastatin significantly increased density of bony defects in rat mandibles.

Statins, including simvastatin, showed biologically significant anti-oxidant, ${ }^{15}$ anti-inflammatory ${ }^{16}$ and anabolic effects on osteoblastic bone formation which could prove beneficial in the field of orthodontics. Han and his colleagues ${ }^{17}$ revealed that the systemic administration of simvastatin could minimize post-orthodontic relapse through inhibition of the bone-resorbing activity of osteoclasts as well as stimulating bone formation. Moreover, the impressive long-term safety profile of simvastatin renders it a suitable agent for use in orthodontic therapy. ${ }^{18}$

This study used an experimental rabbit model to explore the effect of local administration of simvastatin on post-orthodontic relapse. It was hypothesized that the osteoinductive effects of simvastatin on the dental supporting tissues as well as the inhibition of alveolar bone resorption might minimize post-orthodontic relapse. The null hypothesis was that the local administration of simvastatin had no effect on post-orthodontic relapse.

\section{Material and Methods}

The present study was conducted in accordance with ARRIVE guidelines ${ }^{19}$ for conducting animal studies. The study was conducted after the approval of the Ethics Committee, Faculty of Dentistry, Alexandria University.

Calculation of the required sample size was performed using the formula for studies comparing paired continuous data ${ }^{20}$. Based on the results of a previous study yielding a standard deviation of $0.548 \mathrm{~mm},{ }^{21}$ it was determined that a conservative sample size of 9 rabbits would be sufficient to detect an effect size of $0.7 \mathrm{~mm}$ difference in a split-mouth deign at a study power of $90 \%$ and a significance level of 0.05 . However, allowing for $10 \%$ expected attrition; ${ }^{22}$ sample size was corrected to a total of 10 rabbits.

Ten 16-week-old healthy male white New Zealand (Oryctolagus Cuniculus) rabbits were included in the study. The included rabbits had a body weight between $2 \mathrm{~kg}$ and $3 \mathrm{~kg}$ with normal development of dentition and the presence of mandibular incisors, premolars and molars. All experimental procedures were performed under general anesthesia by intramuscular injection of Ketamine (Ketamine Alfasan 10\%, Alfasan Group of Companies, Woerden, The Netherlands) and Xylazine (Xyla-ject Injectable Solution, ADWA CO. S.A.E, $10^{\text {th }}$ of Ramadan City, Egypt). 
Rabbits were kept under standardized conditions of light and dark schedule at the animal house of Alexandria Medical Research Institute. Animals had free access to a soft standard nutritional diet to minimize the incidence of appliance breakage.

A prospective randomized split-mouth experimental trial was implemented in each rabbit. The appliance used to promote tooth movement was similar to the model described by Pithon and Ruellas. All rabbits received an orthodontic appliance consisting of a $13 \mathrm{~mm}$ nickel titanium closed coil spring (Nickel Titanium Closed Coil Springs, 13mm, Light, Jiscop Co. LTD, Gunpo-si, Gyeonggi-do, Korea) stretched between the mandibular first premolars and mandibular incisors bilaterally. The activation force of the spring was measured with a force gauge (Correx Gauge, Haag-Streit, Koeniz Switzerland) such that the spring could provide a force of $80 \mathrm{cN}$. A ligature wire was used to encircle the mandibular second premolar, first molar and second molar and tied in an attempt to stabilize them in position and minimize the influence of interseptal fibers. The appliance was left in place for 21 days to achieve appreciable tooth movement.

Figure 1. Lateral view of experimental orthodontic appliance in situ.

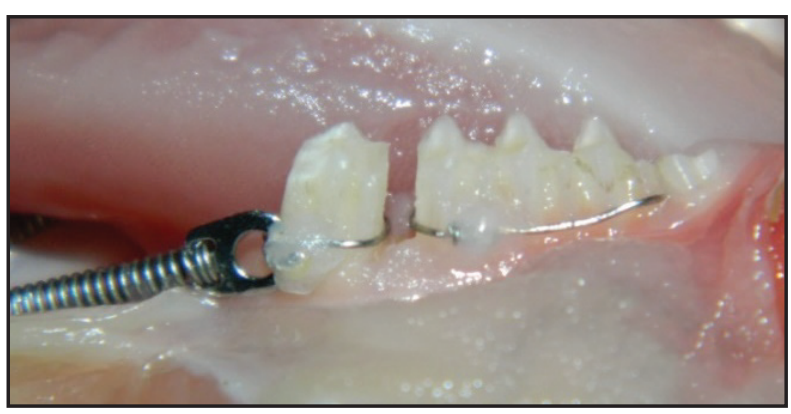

At the end of the active tooth movement, orthodontic appliances were removed and the experimental teeth were allowed to relapse for further 21 days. Each mandibular quadrant was randomly assigned to one of the experimental groups; control group (A) where teeth were allowed to relapse with the local injection of control vehicle solution on days 21, 28 and 35 and simvastatin group (B) where teeth were allowed to relapse with the local injection of simvastatin solution on days 21, 28 and 35 . The assignment of each mandibular quadrantto one of the experimental groups was performed using a computer generated list of random numbers. The individual generating the randomization list was blinded to the treatment groups.

Simvastatin powder (Simvastatin PHR1438, Sigma-Aldrich Co., St. Louis, MO, USA) was prepared at a concentration of $0.5 \mathrm{mg} / 180 \mu \mathrm{l}$ solution. Pluronic F-127 (Pluronic F127, Sigma-Aldrich Co., St. Louis, MO, USA) acted as the carrier for the simvastatin in group B. Pluronic control vehicle solution without simvastatin was administrated in group A.

Intraligamentous injection was performed using an intraligamentous injector (Saniject, Saniswiss Sa Co., Genève, Switzerland) into the distal periodontal space of mandibular first premolar delivering $180 \mu 1$ of solution. The solutions were injected by an operator blinded to the contents of the syringes.

Impressions of experimental teeth were performed on the $21^{\text {st }}$ and $42^{\text {th }}$ day using injection type silicone vinyl polysiloxane impression material (3M ESPE Express Vinyl Polysiloxane Impression Material-Fast set, 3M ESPE Dental Products, St. Paul, MO, USA) loaded into previously fabricated custom trays. The impressions were then poured using an improved die stone (Elite Rock Dental Stone, Zhermack S.P.A, Badia Polesine, Rovigo, Italy). Magnitudes of tooth movement and postorthodontic relapse as well as relapse percentage were calculated on casts of the experimental 
teeth using incremental thickness gauges (OEM Feeler Gauge 25345, OEM Company, Mineola, NY, USA).

Reliability of Measurements of Tooth Movement and Relapse

Measurements were performed in a blinded fashion by a single investigator. The intra- examiner errors for tooth movement measurements were assessed by repeating the measurements of 30 randomly selected 3D models, 2 weeks apart by the same investigator. Intraclass correlation coefficient was calculated to assess intra-rater reliability. Bland Altman analysis was conducted to quantify the level of the agreement between the two repeated measurements.

Data Management and Statistical Analysis

Statistical Package for Social Sciences Software (IBM SPSS Statistics for Windows, Version 23, IBM Corp., Armonk, NY, USA) was used for conducting statistical analysis.
Kolmogorov-Smirnov and Shapiro-Wilk tests were conducted to assess the normality assumption. Once verified, Paired Samples t-Test was conducted to compare mean values between different groups.

\section{Results}

Three experimental rabbits failed to survive and were excluded from the study. Since the percentage of attrition was higher than the estimated percentage, two rabbits fulfilling the eligibility criteria were procured and the same experimental procedures were performed to satisfy the required sample size of 9 rabbits per group. The rest of the animals tolerated the experimental procedures well, with no discernible effects on overall health. Tooth Movement and Relapse

Mean values and standard deviations calculated for the magnitudes of tooth movement and post-orthodontic relapse as well as relapse percentage in groups A and B are summarized in Table I.

Table I. Magnitudes of tooth movement and relapse, and percentage of relapse in control group A and simvastatin group B.

\begin{tabular}{|c|c|c|c|}
\hline & $\begin{array}{c}\text { Magnitude of } \\
\text { Movement }^{\mathrm{a}}\end{array}$ & $\begin{array}{c}\text { Magnitude of } \\
\text { Relapse }^{\mathrm{a}}\end{array}$ & $\begin{array}{c}\text { Relapse } \\
\text { Percentage }^{\mathrm{b}}\end{array}$ \\
\hline Group A & $1.72 \pm 0.54$ & $1.31 \pm 0.53$ & $76.16 \pm 19.18$ \\
\hline Group B & $1.63 \pm 0.64$ & $1.14 \pm 0.47$ & $69.93 \pm 27.31$ \\
\hline
\end{tabular}

a Data presented in $\mathrm{mm}($ Mean $\pm \mathrm{SD})$

$\mathrm{b}$ Data presented as percentage of corresponding tooth movement (Mean $\pm \mathrm{SD}$ )

In group $\mathrm{A}$, the magnitude of tooth movement was $1.72 \pm 0.54 \mathrm{~mm}$, while the magnitude of post-orthodontic relapse was $1.31 \pm 0.53 \mathrm{~mm}$ yielding a relapse percentage of $76.16 \pm 19.18 \%$ of the achieved tooth movement.
In group $\mathrm{B}$, the magnitude of tooth movement was $1.63 \pm 0.64 \mathrm{~mm}$, while the magnitude of post-orthodontic relapse was $1.14 \pm 0.47 \mathrm{~mm}$ yielding a relapse percentage of $69.93 \pm 27.31 \%$ of the achieved tooth movement. 
Results of Kolmogorov-Smirnov and Shapiro-Wilk normality tests for magnitudes of tooth movement and post-orthodontic relapse as well as relapse percentage confirmed the assumption of normality.

Results of paired samples t-test revealed the absence of a significant difference between magnitudes of tooth movement achieved in groups $\mathrm{A}$ and $\mathrm{B}(\mathrm{P}=0.738)$.

Comparing the relapse magnitudes between the 2 groups, results of paired samples t-test revealed no significant difference between magnitudes of post-orthodontic relapse in groups $A$ and $B(P=0.458)$. Similarly, no significant difference was reported in the percentage of post-orthodontic relapse between groups A and $\mathrm{B}(\mathrm{P}=0.562)$.

Reliability of Measurements of Tooth Movement and Relapse

The estimated intra-class correlation coefficient for the repeated measurements of tooth movement was 0.91 revealing a strong evidence for the reliability of the performed measurements. Bland-Altman analysis was performed with a resulting coefficient of repeatability of $0.04 \mathrm{~mm}$ showing high level of agreement for most of the measurements excluding the presence of fixed or systematic bias.

\section{Discussion}

An animal model was selected to test the hypothesis of the present study. Rats and rabbits are considered the ideal animals to obtain a clear picture of bony changes under stress. ${ }^{24}$ Furthermore, rabbits have sufficient periodontal width for the intraligamentous injection required for the present study. ${ }^{25}$ The processes involved in bone remodeling in humans and rodents could be considered almost similar due to the similarity of the morphology and function of cells involved in bone remodeling. In comparison to other species, such as primates and some rodents, the rabbit model has faster bone turnover. ${ }^{26}$ Though Mundy et a19 reported on a quick BMP-2 response to statins within 3-5 days in vivo and in vitro, beneficial effects of statins on fracture healing were extended to 5-14 days of local statin exposure to the fracture site, ${ }^{27}$ suggesting that statins cause a delayed onset of endogenous BMP-2 production. ${ }^{28}$ Thus, extending the experimental tooth movement phase in the present study to 21 days allowed a sufficient considerable time for the effect of simvastatin on the periodontium.This period of active tooth movement was in accordance with previous studies evaluating tooth movements in rabbits..$^{29,30}$

The local simvastatin dosage in the present study was selected based on previous animal studies recommending a dose of $0.1-0.5 \mathrm{mg}$ of simvastatin as the optimal local dose for stimulating maximum bone regeneration without inducing local inflammation. ${ }^{31}$

Pluronic F127 exhibits the unique property of reversible thermal gelation by transforming from low viscosity solutions at a temperature $\leq 10^{\circ} \mathrm{C}$ to clear semi-solid gels at normal physiological body temperature; above its transitional temperature $\left(21^{\circ} \mathrm{C}\right)$. Moreover, the drug release from such a gel occurs over a period of up to one week. ${ }^{32}$ These properties rendered Pluronic F127 an attractive vehicle for controlled release.

Tooth Movement and Relapse

Results of measurements of tooth movement in both groups A and B revealed no significant difference in the magnitude of tooth movement achieved at the end of the experimental tooth movement phase. This finding was expected due to the use of the same experimental orthodontic appliance with nearly the same force magnitude within the same 
study settings. In both groups $\mathrm{A}$ and $\mathrm{B}$, the experimental teeth showed post-orthodontic relapse towards their original positions after the removal of the orthodontic appliance. Despite the relapse magnitude and percentage in group B being less than those reported for group A, the differences were not statistically significant. This post-orthodontic relapse was expected because in this initial period upon appliance removal, the tooth undergoes a rebound shift within the tooth socket. ${ }^{33}$

On the other hand, results of postorthodontic relapse in the present study agree with the findings reported by Vieira et al ${ }^{34}$ demonstrating that the systemic administration of simvastatin did not inhibit relapse of induced tooth movement in rats. However, this contradicts the results of Han et al 17 showing a reduction in the relapse distances after systemic administration of simvastatin.

Though the percentage of postorthodontic relapse in the experimental quadrants receiving simvastatin administration was reduced, the reduction was not statistically significant compared to the corresponding quadrants receiving control vehicle solutions.

Though the causal mechanisms of orthodontic relapse remain relatively unclear, it would seem to be a complex multifactorial process with the difficulty of stressing a single causative factor. Despite the fact that remodeling of the periodontal ligament and surrounding alveolar bone is an important element in the relapse process $,{ }^{35},{ }^{36}$ other potential factors may include normalization of the periodontal vasculature following orthodontic force, ${ }^{37}$ increase in elasticity of the gingiva that is being retracted and compressed in the direction of the tooth movement, ${ }^{38}$ and stretching of transseptal fibers. ${ }^{33,39}$ This reflected the clinical observation of the evident relapse in both groups A and B in the present study, in which the experimentally moved teeth had relapsed back towards their original positions. Possibly, relapse energy stored in the collagenous periodontal and transseptal fiber systems was gradually released once the orthodontic appliance was removed, resulting in significant post-orthodontic relapse. ${ }^{40}$

\section{Conclusion}

Local simvastatin administration could not minimize post-orthodontic relapse magnitude to a significant level.

\section{References}

1. Sadowsky C, Schneider BJ, BeGole EA, Tahir E. Long-term stability after orthodontic treatment: nonextraction with prolonged retention. Am J Orthod Dentofacial Orthop 1994;106(3):243-9.

2. Kahl-Nieke B, Fischbach H, Schwarze CW. Post-retention crowding and incisor irregularity: a long-term follow-up evaluation of stability and relapse. Br J Orthod 1995;22(3):249-57.

3. Hudson JB, Hatch N, Hayami T, Shin JM, Stolina M, Kostenuik PJ, et al. Local delivery of recombinant osteoprotegerin enhances post-orthodontic tooth stability. Calcif Tissue Int 2012;90(4):330-42.

4. Kim TW, Yoshida Y, Yokoya K, Sasaki T. An ultrastructural study of the effects of bisphosphonate administration on osteoclastic bone resorption during relapse of experimentally moved rat molars. Am J Orthod Dentofacial Orthop 1999;115(6):645-53.

5. Zhao N, Lin J, Kanzaki H, Ni J, Chen Z, Liang $\mathrm{W}$, et al. Local osteoprotegerin gene transfer inhibits relapse of orthodontic tooth movement. Am J Orthod Dentofacial Orthop 2012;141(1):30-40. 
6. Hirate Y, Yamaguchi M, Kasai K. Effects of relaxin on relapse and periodontal tissue remodeling after experimental tooth movement in rats. Connect Tissue Res 2012;53(3):207-19.

7. Hassan AH, Al-Hubail A, Al-Fraidi AA. Bone inductive proteins to enhance post-orthodontic stability. Angle Orthod 2010;80(6):1051-60.

8. Edwards CJ, Russell RG, Spector TD. Statins and bone: myth or reality? Calcif Tissue Int 2001;69(2):63-6.

9. Mundy G, Garrett R, Harris S, Chan J, Chen D, Rossini G, et al. Stimulation of bone formation in vitro and in rodents by statins. Science 1999;286(5446):1946-9.

10. Maeda T, Matsunuma A, Kawane T, Horiuchi N. Simvastatin promotes osteoblast differentiation and mineralization in MC3T3-E1 cells. Biochem Biophys Res Commun 2001;280(3):874-7.

11. Kinra P, Khan S2. Simvastatin: Its potential new role in periodontal regeneration. Biology and Medicine 2011;3(2):215-21.

12. Yazawa H, Zimmermann B, Asami Y, Bernimoulin JP. Simvastatin promotes cell metabolism, proliferation, and osteoblastic differentiation in human periodontal ligament cells. J Periodontol 2005;76(2):295-302

13. Lee Y, Schmid MJ, Marx DB, Beatty MW, Cullen DM, Collins ME, et al. The effect of local simvastatin delivery strategies on mandibular bone formation in vivo. Biomaterials 2008;29(12):1940-9.

14. Ozec I, Kilic E, Gumus C, Goze F. Effect of local simvastatin application on mandibular defects. J Craniofac Surg 2007;18(3):546-50.
15. Giroux LM, Davignon J, Naruszewicz M. Simvastatin inhibits the oxidation of low- density lipoproteins by activated human monocyte- derived macrophages. Biochim Biophys Acta 1993;1165(3):335-8.

16. Davignon J, Laaksonen R. Low-density lipoprotein-independent effects of statins. Curr Opin Lipidol 1999;10(6):543-59.

17. Han G, Chen Y, Hou J, Liu C, Chen C, Zhuang $\mathrm{J}$, et al. Effects of simvastatin on relapse and remodeling of periodontal tissues after tooth movement in rats. Am J Orthod Dentofacial Orthop 2010;138(5):550 e1-7; discussion 50-1.

18. Guthrie RM, 2006. How safe is aggressive statin therapy? Prog Cardiovasc Nurs 2006;21(3):140-5.

19. Kilkenny C, Browne WJ, Cuthill IC, Emerson M, Altman DG. Improving bioscience research reporting: the ARRIVE guidelines for reporting animal research. PloS Biol 2010;8(6):e1000412.

20. Festing MF, Altman DG. Guidelines for the design and statistical analysis of experiments using laboratory animals. ILAR J. 2002;43(4):244- 58.

21. Venkataramana V, Chidambaram S, Reddy BV, Goud EV, Arafath M, Krishnan S. Impact of Bisphosphonate on Orthodontic tooth movement and osteoclastic count: An Animal Study. J Int Oral Health 2014;6(2):1-8.

22. Jones SR, Carley S, Harrison M. An introduction to power and sample size estimation. Emerg Med J 2003;20(5):453-8.

23. Pithon MM, Ruellas ACO. Histological evaluation of the phenobarbital (Gardenal ${ }^{\mathrm{TM}}$ ) influence on orthodontic movement: a study in rabbits. Dental Press J Orthod 2011;16(4):47-54. 24. Storey E. Tissue response to the movement 
of bones. Am J Orthod 1973;64(3):229-47.

25. Caglaroglu M, Erdem A. Histopathologic investigation of the effects of prostaglandin E2 administered by different methods on tooth movement and bone metabolism. Korean J Orthod 2012;42(3):118-28.

26. Castaneda S, Largo R, Calvo E, Rodriguez-Salvanes F, Marcos ME, DiazCuriel M, et al. Bone mineral measurements of subchondral and trabecular bone in healthy and osteoporotic rabbits. Skeletal Radiol 2006;35(1):34-41.

27. Wang JW, Xu SW, Yang DS, Lv RK. Locally applied simvastatin promotes fracture healing in ovariectomized rat. Osteoporos Int 2007;18(12):1641-50.

28. Pauly S, Luttosch F, Morawski M, Haas NP, Schmidmaier G, Wildemann B. Simvastatin locally applied from a biodegradable coating of osteosynthetic implants improves fracture healing comparable to BMP-2 application. Bone 2009;45(3):505-11.

29. Roche JJ, Cisneros GJ, Acs G. The effect of acetaminophen on tooth movement in rabbits. Angle Orthod 1997;67(3):231-6.

30. Kilic N, Oktay H, Ersoz M. Effects of force magnitude on tooth movement: an experimental study in rabbits. Eur J Orthod 2010;32(2):154-8.

31. Chen S, Yang JY, Zhang SY, Feng L, Ren J. Effects of simvastatin gel on bone regeneration in alveolar defects in miniature pigs. Chin Med J (Engl) 2011;124(23):3953-8.

32. Kabanov AV, Batrakova EV, Alakhov VY. Pluronic block copolymers as novel polymer therapeutics for drug and gene delivery. J Control Release 2002;82(2):189-212.

33. Reitan K. Clinical and histologic observations on tooth movement during and after orthodontic treatment. Am J Orthod 1967;53(10):721-45.

34. Vieira GM, Chaves SB, Ferreira VM, Freitas KM, Amorim RF. The effect of simvastatin on relapse of tooth movement and bone mineral density in rats measured by a new method using microtomography. Acta Cir Bras 2015;30(5):319-27.

35. Franzen TJ, Brudvik P, Vandevska-Radunovic V. Periodontal tissue reaction during orthodontic relapse in rat molars. Eur J Orthod 2013;35(2):152-9.

36. Franzen TJ, Monjo M, Rubert M, Vandevska-Radunovic V. Expression of bone markers and micro-CT analysis of alveolar bone during orthodontic relapse. Orthod Craniofac Res 2014;17(4):249-58.

37. Murrell EF, Yen EH, Johnson RB. Vascular changes in the periodontal ligament after removal of orthodontic forces. Am J Orthod Dentofacial Orthop 1996;110(3):280-6.

38. Redlich M, Shoshan S, Palmon A. Gingival response to orthodontic force. Am J Orthod Dentofacial Orthop 1999;116(2):152-8.

39. Parker GR. Transseptal fibers and relapse following bodily retraction of teeth: a histologic study. Am J Orthod 1972;61:331-44.

40. Van Leeuwen EJ, Maltha JC, Kuijpers-Jagtman $A M$, van ' $t$ Hof MA. The effect of retention on orthodontic relapse after the use of small continuous or discontinuousforces. An experimental study in beagle dogs. Eur J Oral Sci 2003;111(2):111-6. 